\title{
Acute pericarditis: Update on diagnosis and management
}

\author{
Author: Tevfik F Ismail ${ }^{A}$
}

Acute pericarditis accounts for $\sim 5 \%$ of presentations with acute chest pain. Tuberculosis is an important cause in the developing world, however, in the UK and other developed settings, most cases are idiopathic/viral in origin. Nonsteroidal anti-inflammatory drugs (NSAIDs) remain the cornerstone of treatment. At least one in four patients are at risk of recurrence. The addition of 3 months of colchicine can more than halve the risk of this (number needed to treat $=$ four). Low-dose steroids can be helpful second-line agents for managing recurrences as adjuncts to NSAIDs and colchicine but should not be used as first-line agents. For patients failing this approach and/or dependent on corticosteroids, the interleukin-1 $\beta$ antagonist anakinra is a promising option, and for the few patients who are refractory to medical therapy, surgical pericardiectomy can be considered. The long-term prognosis is good with $<0.5 \%$ risk of constriction for patients with idiopathic acute pericarditis.

\section{Introduction}

The pericardial sac is made up of an inner mesothelial layer which covers the heart (visceral) and lines an outer fibrous layer onto which the mesothelium reflects (parietal layer). It produces up to $50 \mathrm{~mL}$ of fluid which serves to lubricate the motion of the heart, and overall serves to prevent excessive cardiac motion and anchor it in the mediastinum. Pericardial disease results from inflammation of the pericardium, which in turn can give rise to an effusion; and rigidity of the pericardium giving rise to the constriction syndrome. The visceral pericardium is innervated by branches of the sympathetic trunk which carry afferent pain fibres in a cardiac distribution and the vagus which may trigger vagally mediated reflexes in acute pericarditis. In contrast, the parietal and fibrous pericardium are innervated by somatosensory branches of the phrenic nerve which can give rise to referred pain to the shoulder.

\section{Diagnosis and initial investigation}

Pericarditis is a relatively common cause of chest pain accounting for $\sim 5 \%$ of all chest pain admissions. ${ }^{1}$ In the UK, the majority of cases are idiopathic and likely viral in origin, in contrast to

Author: ${ }^{\text {A }}$ linical senior lecturer and consultant cardiologist, King's College London and Guy's and St Thomas' NHS Foundation Trust, London, UK the situation in the developing world where tuberculosis is a common cause. ${ }^{2}$ Patients typically complain of chest pain which is central, worse with inspiration or when lying down and improved by sitting up/forward. Auscultation may reveal a characteristic pericardial friction rub, although this can be evanescent and can require repeated evaluation for detection. ${ }^{3}$ Electrocardiography (ECG) classically reveals widespread saddle-shaped ST elevation with associated PR-depression and is helpful for excluding other causes of chest pain. Chest $X$-ray is frequently normal unless there is a sizeable pericardial effusion. Inflammatory markers (erythrocyte sedimentation rate and C-reactive protein) are often raised and there may also be slight elevations of troponin if there is associated myopericarditis. More significant elevations and/or clinical or echo features of left ventricular dysfunction should prompt a consideration of myocarditis instead or so-called perimyocarditis where myocardial involvement predominates.

\section{Key points}

Diagnosis of acute pericarditis requires the presence of any two of:

$>$ pericarditic chest pain

$>$ pericardial rub

> saddle-shaped ST-elevation and/or PR-depression

$>$ non-trivial new or worsening pericardial effusion.

Most cases in the UK are idiopathic (presumed viral) and can be managed as an outpatient in the absence of any red-flag features or myopericarditis.

Inpatient investigation and more intensive evaluation for a non-viral aetiology should be considered where there is any fever $>38^{\circ} \mathrm{C}$; gradual onset; large effusion ( $>20 \mathrm{~mm}$ ) or tamponade; lack of response to 1 week of non-steroidal antiinflammatory drugs or where there is any history of trauma, immunosuppression/deficiency or oral anticoagulant use.

Colchicine at $500 \mu \mathrm{g}$ twice per day (if $>70 \mathrm{~kg}$ ) or $500 \mu \mathrm{g}$ once per day (if $<70 \mathrm{~kg}$ ) for 3 months more than halves the risk of recurrence (number needed to treat $=$ four).

Corticosteroids should not be used as first-line agents for idiopathic acute pericarditis but may have a role as adjunctive therapy for cases of recurrent disease and where there is an underlying autoimmune rheumatic disease.

KEYWORDS: Pericarditis, colchicine, chest pain, pericardiectomy, constriction 
Fig 1. Cardiovascular magnetic resonance in acute pericarditis. a)

T2-weighted spin echo image depicting the acutely inflamed pericardium which appears high signal (arrow). There are also associated bilateral pleural effusions. b) Late gadolinium enhancement sequence revealing avid uptake of contrast by the inflamed pericardium (arrow). Healthy pericardium does not enhance with contrast.

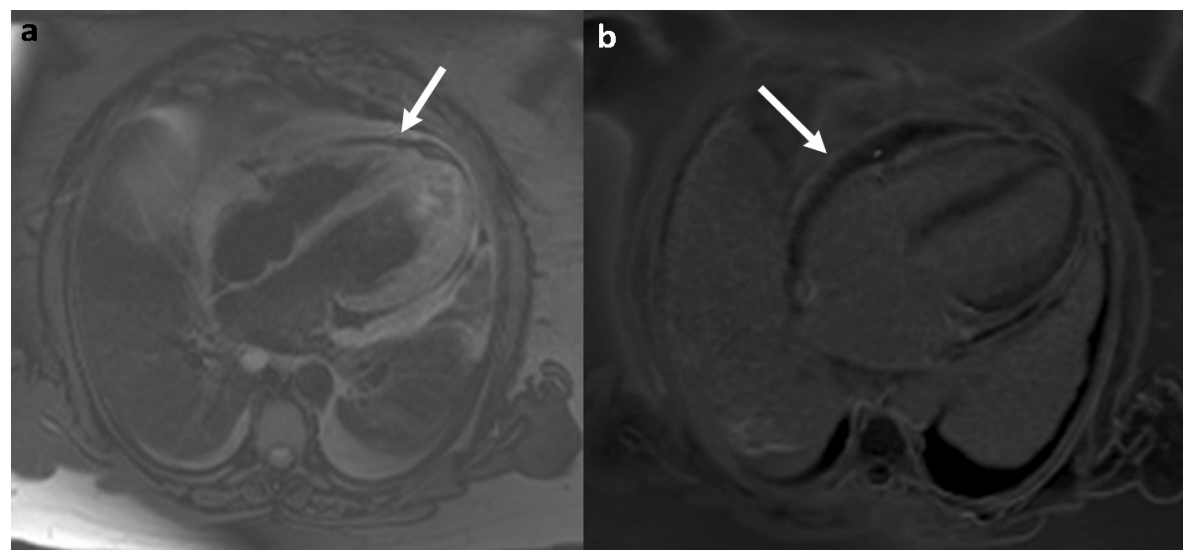

Diagnosis of pericarditis requires the presence of two of typical pericardial chest pain; pericardial friction rub; widespread STelevation and/or PR-depression; and a new or increasing nontrivial pericardial effusion. ${ }^{2}$ If diagnostic uncertainty remains, cardiovascular magnetic resonance with T2-weighted and late gadolinium enhancement imaging can be helpful for confirming the presence of any pericardial inflammation, and excluding concomitant myocarditis as well as other differentials (Fig 1). ${ }^{4}$ The majority of cases resolve within a month and the yield of investigation for a precipitant, in particular viral serology, is low and generally not recommended. ${ }^{2}$ Pericarditis persisting for more than 4-6 weeks but less than 3 months is termed incessant. Pericarditis persisting longer than 3 months is termed chronic. If there is a period of intervening remission lasting more than 4-6 weeks, the term recurrent is used. These terms are relevant to therapeutic decision making and investigation pathways.

\section{Risk stratification}

A thorough history is a vital step in risk stratification (Box 1). A number of clinical features may portend an increased risk of complications and indicate the need for initial inpatient investigations and an active search for an aetiology: fever $>38^{\circ} \mathrm{C}$; gradual onset of symptoms; presence of a large effusion ( $>20 \mathrm{~mm}$ ) or features of tamponade physiology; and lack of response to treatment after 1 week of anti-inflammatories. ${ }^{5}$ The presence of myopericarditis, a history of trauma, use of oral anticoagulants and a background of immunosuppression are considered minor adverse prognostic markers which may also trigger inpatient evaluation. ${ }^{2}$

\section{Treatment}

For all patients, exercise restriction is recommended by current guidelines for the duration of symptoms and for at least 3 months in athletes, although this is based on expert consensus opinion rather than sound evidence. ${ }^{2,6}$ Acute pericarditis can be treated with a non-steroidal anti-inflammatory drug (NSAID) such as ibuprofen at $600 \mathrm{mg}$ three times per day (tds) for 1-2 weeks usually with a proton pump inhibitor, tapering once inflammatory markers have normalised typically by $\sim 400 \mathrm{mg}$ per week. ${ }^{2}$ If there are any significant risk factors for or any history of coronary disease, aspirin may be preferred to ibuprofen at a dose of $900 \mathrm{mg}$ tds for $1-2$ weeks, tapering by $\sim 600 \mathrm{mg}$ per week thereafter assuming symptoms resolve, and inflammatory markers normalise. Pericarditis can recur in up to $30 \%$ of patients within 1.5 years, and in $\sim 55 \%$ of those with a previous recurrence. ${ }^{7,8}$ The adjunctive use of colchicine in acute pericarditis for 3 months at a dose of $500 \mu \mathrm{g}$ twice per day (bd) for those $>70 \mathrm{~kg}$ and $500 \mu \mathrm{g}$ once per day if $<70 \mathrm{~kg}$ (Table 1), increases the rate of remission at 1 week and importantly, more than halves the risk of recurrent or

\section{Box 1. History-taking for the clinical evaluation of acute pericarditis}

Chest pain: central, worse lying down, or with inspiration and better sitting up/forward.

Palpitations/tachycardia (may suggest underlying myocarditis/myocardial involvement or presence of haemodynamically significant effusion).

Pre-syncope/syncope (may suggest significant associated effusion and/or myocardial involvement).

Pleuritic symptoms (may suggest concomitant pleuritis, connective tissue disease or periodic fever syndrome).

Hiccups (phrenic nerve irritation from a large pericardial effusion).

Dysphagia (oesophageal compression by large effusion; symptom of underlying malignancy or scleroderma).

Fever (may point towards bacterial infection, haematological malignancy, systemic autoimmune disease, periodic fever syndromes).

Weight loss (neoplasia, tuberculosis, systemic autoimmune disease).

Past history of cardiac surgery/thoracic trauma, autoimmune rheumatic disease, immunodeficiency.

Drugs: causative drugs (eg hydralazine), immunosuppression, oral anticoagulants.

Family history: autoimmune disease, hereditary periodic fever syndrome/recurrent pericarditis. 
Table 1. Practical prescribing of colchicine for acute pericarditis

\begin{tabular}{|c|c|c|}
\hline Patient weight & $<70 \mathrm{~kg}$ & $>70 \mathrm{~kg}$ \\
\hline Standard dosing & $\begin{array}{l}500 \mu \mathrm{g} \text { once per } \\
\text { day for } 3 / 12\end{array}$ & $500 \mu \mathrm{g}$ twice per day for $3 / 12$ \\
\hline Dose if gastrointestinal intolerance develops ${ }^{a}$ & $\begin{array}{l}250 \mu \mathrm{g} \text { once per } \\
\text { day or } 500 \mu \mathrm{g} \text { on } \\
\text { alternate days }\end{array}$ & $500 \mu \mathrm{g}$ once per day \\
\hline Cautions & \multicolumn{2}{|l|}{ Contraindications } \\
\hline Baseline blood abnormalities, especially leucopenia & \multicolumn{2}{|c|}{ Pregnancy (advise use of effective contraception) } \\
\hline Liver impairment & \multicolumn{2}{|l|}{ Breastfeeding } \\
\hline Renal impairment & \multicolumn{2}{|l|}{ Blood dyscrasias } \\
\hline $\begin{array}{l}\text { For eGFR } 15-30 \mathrm{~mL} / \mathrm{min} / 1.73 \mathrm{~m}^{2} \text { dose } 500 \mu \mathrm{g} \text { every } 2-3 \text { days with caution and } \\
\text { close monitoring; for moderate renal impairment }\left(30-60 \mathrm{~mL} / \mathrm{min} / 1.73 \mathrm{~m}^{2} \text { ), }\right.\end{array}$ & \multicolumn{2}{|c|}{$\begin{array}{l}\text { Severe renal impairment (eGFR }<15 \mathrm{~mL} / \mathrm{min} / \\
1.73 \mathrm{~m}^{2} \text { ) - avoid }\end{array}$} \\
\hline reduce dose/interval as for gastrointestinal intolerance & \multicolumn{2}{|c|}{ Dialysis - avoid, not removed by dialysis } \\
\hline Gastrointestinal disorders & \multicolumn{2}{|c|}{ Severe hepatic impairment } \\
\hline Cardiovascular disease & \multirow{2}{*}{\multicolumn{2}{|c|}{$\begin{array}{l}\text { Concomitant therapy with potent CYP3A4 inhibitor } \\
\text { or P-glycoprotein inhibitor, especially if concomitant } \\
\text { renal or hepatic impairment. }\end{array}$}} \\
\hline Elderly/frail patients & & \\
\hline \multicolumn{3}{|l|}{ Interactions (which can significantly boost drug levels) } \\
\hline P-glycoprotein inhibitors & \multicolumn{2}{|l|}{ CYP3A4 inhibitors } \\
\hline Azithromycin & \multicolumn{2}{|c|}{ Macrolides eg clarithromycin } \\
\hline Verapamil & \multirow{2}{*}{\multicolumn{2}{|c|}{$\begin{array}{l}\text { Triazole antifungals eg itraconazole, ketoconazole, } \\
\text { posaconazole }\end{array}$}} \\
\hline Diltiazem & & \\
\hline Dihydropyridines & \multicolumn{2}{|c|}{ HIV-protease inhibitors eg ritonavir } \\
\hline Quinidine & \multicolumn{2}{|c|}{ Other significant interactions } \\
\hline Amiodarone & \multirow{2}{*}{\multicolumn{2}{|c|}{ Statins and fibrates (risk of rhabdomyolysis) }} \\
\hline Ciclosporin & & \\
\hline \multicolumn{3}{|l|}{ Proton pump-inhibitors } \\
\hline \multicolumn{3}{|l|}{ Paroxetine } \\
\hline Sertraline & & \\
\hline
\end{tabular}

${ }^{a}=$ Colchicine may induce a degree of acquired lactose intolerance which may in part be responsible for diarrhoea. ${ }^{9}$ A lactose-free diet may help some patients together with dose reduction as advised above. Lactose is also a common excipient. Colchicine may also impair vitamin B12 absorption; eGFR = estimated glomerular filtration rate.

incessant pericarditis (number needed to treat $=$ four). ${ }^{7}$ Colchicine while safe if appropriately used has a narrow therapeutic index and therefore extra attention should be paid when prescribing to potential drug interactions and comorbidities that may influence the pharmacokinetics of the drug (Table 1).

Corticosteroids should be avoided if possible unless there is a clear underlying autoimmune rheumatic disease or a contraindication to NSAIDs/colchicine, eg pregnancy. While initially effective, the use of steroids may promote recurrence and may attenuate the efficacy of colchicine if used first-line. ${ }^{10}$ The reasons for this are unclear but it is thought that most cases of idiopathic acute pericarditis are viral in origin and that steroid use may promote viral replication and delay clearance thereby maintaining the trigger for inflammation. ${ }^{11}$ For idiopathic pericarditis, they should only be used adjunctively after a trial of NSAIDs and colchicine for patients with recurrent disease. Doses higher than $0.2-0.5 \mathrm{mg} / \mathrm{kg} /$ day of prednisolone are not required and incur increased risk of side effects without additional efficacy. ${ }^{12}$ Treatment is continued for 4 weeks and if symptoms resolve and inflammatory markers normalise, doses are tapered by $5-10 \mathrm{mg} /$ day every week until $25 \mathrm{mg}$ is reached and thereafter by $2.5 \mathrm{mg} /$ day every 2 weeks until $15 \mathrm{mg}$ is reached. The risk of recrudescence of symptoms is highest below $15 \mathrm{mg}$ so thereafter, the taper is slowed to $1-2.5 \mathrm{mg} /$ day every $2-6$ weeks. When tapering away medication, colchicine should be the last agent to be withdrawn. ${ }^{2}$ Recurrences may manifest with chest pain alone without other cardinal features of pericarditis, particularly where inflammation has only been partially treated..$^{13}$ In this setting, cardiovascular magnetic resonance can be of value to confirm ongoing inflammation. ${ }^{13}$

For patients failing to obtain remission with first-line (NSAID plus colchicine) or second-line approaches (NSAID plus steroid plus colchicine), third-line therapeutic options include azathioprine, intravenous immunoglobulin and anakinra (an interleukin-1 $\beta$ antagonist). Azathioprine is best used as a steroid-sparing agent (typically at $1-3 \mathrm{mg} / \mathrm{kg} /$ day) and can take up to 3 months to be 
effective. $^{2}$ The effect of intravenous immunoglobulin is more immediate, but its availability is restricted and the evidence base for its use is limited to isolated case reports and small case-series. Nevertheless, allowing for the possibility of publication bias, it appears to have high-efficacy with an excellent safety profile. ${ }^{14}$ There are some initial promising data for anakinra $(2 \mathrm{mg} / \mathrm{kg} / \mathrm{day}$ up to $100 \mathrm{mg}$ ) in patients with $>3$ recurrences, raised inflammatory markers, colchicine-resistance and steroid-dependence. ${ }^{15}$ This may be in keeping with the cardinal role that interleukin-1 plays in the innate immune response as exemplified by patients with hereditary autoinflammatory syndromes. ${ }^{13}$ For patients failing third-line therapeutic options, surgical pericardiectomy is the treatment of last resort although seldom required in clinical practice and usually more often necessary for patients with a previous history of cardiac surgery and/or features of constriction rather than for otherwise uncomplicated idiopathic recurrent pericarditis. ${ }^{16}$

\section{Prognosis and complications}

The prognosis of idiopathic acute pericarditis is generally excellent with a very low risk of long-term sequelae such as constriction $(<0.5 \%))^{17,18}$ The likelihood of the latter is related to the aetiology of pericarditis rather than the number of episodes. Constriction presents with signs and symptoms of heart failure (shortness of breath, fatigue, oedema/ascites) but with normal ejection fraction on echo and often normal or minimally elevated brain natriuretic peptide. ${ }^{19,20}$ Constriction is more likely to occur following pericarditis triggered by tuberculosis/bacterial infection, trauma, and cardiac surgery. The prognosis of myopericarditis mirrors that of pericarditis given the large overlap in aetiology, particularly when left ventricular function is preserved.

\section{Conclusions}

Acute pericarditis is a relatively common cause of acute chest pain which can be readily evaluated by thorough history-taking supplemented by ECG and echocardiography. In the developed world, the majority of cases are idiopathic and while the prognosis with respect to the development of adverse sequelae is excellent, acute episodes and recurrences can have a significant impact on patients' quality of life and well-being. While the study of pericardial diseases does not receive the same degree of attention as acute coronary syndromes, there is an emerging evidence base for the treatment of idiopathic acute pericarditis. The use of colchicine significantly reduces the chances of recurrence but despite this, a troublesome subset of patients remain who experience multiple recurrences. For this subgroup, there is some promising initial data for the interleukin-1 $\beta$ antagonist anakinra. Nevertheless, our understanding of the immunopathology and pathogenesis of this pericardial syndrome remains incomplete and more work is required to address this if further therapeutic advances are to be made.

\section{References}

1 LeWinter MM. Clinical practice. Acute pericarditis. N Engl ] Med 2014;371:2410-6.

2 Adler Y, Charron P, Imazio M et al. 2015 ESC guidelines on the diagnosis and management of pericardial diseases. Eur Heart J 2015:36:2873-4.
3 Khandaker MH, Espinosa RE, Nishimura RA et al. Pericardial disease: Diagnosis and management. Mayo Clin Proc 2010;85: 572-93.

4 Bogaert ], Francone M. Cardiovascular magnetic resonance in pericardial diseases. J Cardiovasc Magn Reson 2009;11:14.

5 Imazio M, Cecchi E, Demichelis B et al. Indicators of poor prognosis of acute pericarditis. Circulation 2007;115:2739-44.

6 Imazio M, Gaita F, LeWinter MM. Evaluation and treatment of pericarditis: A systematic review. JAMA 2015;314:1498-506.

7 Imazio M, Brucato A, Cemin R et al. A randomized trial of colchicine for acute pericarditis. N Engl J Med 2013;369:1522-8.

8 Imazio M, Brucato A, Cemin R et al. Colchicine for recurrent pericarditis (CORP): a randomized trial. Ann Intern Med 2011;155:409-14.

9 Fradkin A, Yahav J, Zemer D, Jonas A. Colchicine-induced lactose malabsorption in patients with familial Mediterranean fever. Isr ] Med Sci 1995;31:616-20.

10 Artom G, Koren-Morag N, Spodick DH et al. Pretreatment with corticosteroids attenuates the efficacy of colchicine in preventing recurrent pericarditis: a multi-centre all-case analysis. Eur Heart J 2005;26:723-7.

11 Brucato A, Maestroni S, Cumetti D et al. Recurrent pericarditis: infectious or autoimmune? Autoimmun Rev 2008;8:44-7.

12 Imazio M, Brucato A, Cumetti D et al. Corticosteroids for recurrent pericarditis: high versus low doses: a nonrandomized observation. Circulation 2008;118:667-71.

13 Cremer PC, Kumar A, Kontzias A et al. Complicated pericarditis: Understanding Risk factors and pathophysiology to inform imaging and treatment. J Am Coll Cardiol 2016;68:2311-28.

14 Imazio M, Lazaros G, Picardi E et al. Intravenous human immunoglobulins for refractory recurrent pericarditis: a systematic review of all published cases. J Cardiovasc Med 2016;17:263-9.

15 Brucato A, Imazio M, Gattorno M et al. Effect of anakinra on recurrent pericarditis among patients with colchicine resistance and corticosteroid dependence: The AIRTRIP randomized clinical trial. JAMA 2016;316:1906-12

16 Khandaker MH, Schaff HV, Greason KL et al. Pericardiectomy vs medical management in patients with relapsing pericarditis. Mayo Clin Proc 2012;87:1062-70.

17 Imazio M, Brucato A, Adler $Y$ et al. Prognosis of idiopathic recurrent pericarditis as determined from previously published reports. Am J Cardiol 2007;100:1026-8.

18 Imazio M, Brucato A, Maestroni S et al. Risk of constrictive pericarditis after acute pericarditis. Circulation 2011;124:1270-5.

19 Leya FS, Arab D, Joyal D et al. The efficacy of brain natriuretic peptide levels in differentiating constrictive pericarditis from restrictive cardiomyopathy. J Am Coll Cardiol 2005;45:1900-2.

20 Ponikowski P, Voors AA, Anker SD et al. 2016 ESC guidelines for the diagnosis and treatment of acute and chronic heart failure: The Task Force for the diagnosis and treatment of acute and chronic heart failure of the European Society of Cardiology (ESC)Developed with the special contribution of the Heart Failure Association (HFA) of the ESC. Eur Heart J 2016:37:2129-200.

Address for correspondence: Dr Tevfik F Ismail, 4th Floor Lambeth Wing, St Thomas' Hospital, Westminster Bridge Road, London SE1 7EH, UK.

Email: tevfik.ismail@kcl.ac.uk 